\title{
Incremental Structure Learning of Three-Layered Gaussian RBF Networks
}

\author{
David Coufal ${ }^{1}$ \\ Institute of Computer Science, Academy of Sciences of the Czech Republic, \\ Pod Vodárenskou věží 2, 18207 Praha 8, Czech Republic \\ coufal@cs.cas.cz
}

\begin{abstract}
In the paper a new incremental algorithm for structure learning of three-layered RBF neural network is proposed. The algorithm is intended to be used for solving high-dimensional learning tasks since they makes troubles to standard structure learning techniques.
\end{abstract}

\section{Introduction}

It is well known that supervised learning of RBF neural networks generally consists of two subtasks named as structure learning and parameters learning. Within structure learning a number of network neurons is determined together with initial setting of network parameters. Parameters learning is then only a suitable readjustment of initially set parameters to network's operation matches given training input/output set as close as possible.

Structure learning is a harder part of a learning process. This fact is well documented by relevant literature offering a variety of different approaches to this task but without such a degree of unification that it is available for parameters learning where we almost always deal with an instance of an optimization problem. In spite of this variety, however, several classes of structure learning algorithms can be recognized. We review in short common of them in the third section of the paper.

As we will see from this review each of presented algorithms suffers from some disadvantage which decreases its applicability. As a reaction to this situation we formulate a new algorithm minimizing presented limitations. The algorithm itself is described within the fourth and the fifth section of the paper. In the sixth section there are presented results of several experiments regarding the algorithm. The paper concludes by the seventh section.

As it is indicated by the title of the paper the new algorithm is considered to be applied on structure learning of three-layered feedforward RBF networks. Especially, we will consider networks with $n$-dimensional input and only onedimensional output. Regarding type of radial basis function we employ Gaussian one but other types of functions can be used as well. In the following section we state an explicit description of a network architecture we are interested in. 


\section{Three-layered Gaussian RBF neural network}

It is well known that a three-layered feedforward network's architecture is given by serial interconnection of an input, a hidden and an output layer, see Fig. 2 . For our case an input layer is considered to consist of $n$ neurons, the hidden layer of $m$ neurons and the output layer only of one neuron.

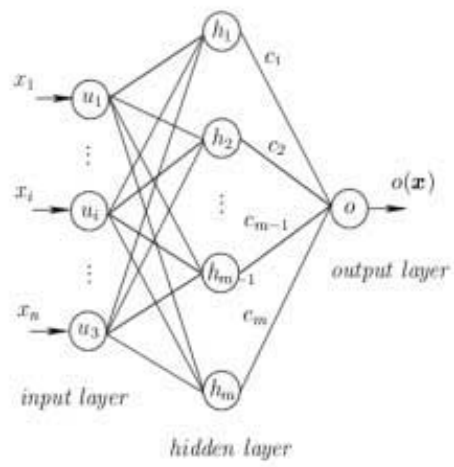

Fig. 1. A three-layered feedforward RBF neural network.

Input neurons are considered to be only transmitting ones. Hidden neurons are considered to be represented by multidimensional Gaussians, hence to act as

$$
h_{j}(\boldsymbol{x})=\exp \left[-\sum_{i=1}^{n} \frac{\left(x_{i}-a_{j i}\right)^{2}}{2 b_{j}^{2}}\right]
$$

where $\boldsymbol{x}$ is an input $\boldsymbol{x}=\left(x_{1}, \ldots, x_{n}\right), \boldsymbol{a}_{j}$ is a central point, $\boldsymbol{a}_{j}=\left(a_{j 1}, \ldots, a_{j n}\right)$, and $b_{j} \neq 0$ is a width parameter. Connections from hidden layer to output layer are endowed by weights $c_{1}, \ldots, c_{m}$. An output neuron then forms a linear combination of outputs of hidden neurons.

Therefore, mathematically, a network's computation can be seen as mapping from $\mathcal{R}^{n}$ to $\mathcal{R}$ given by a linear combination of $m$ multidimensional Gaussians

$$
o(\boldsymbol{x})=\sum_{j=1}^{m} c_{j} \cdot h_{j}(\boldsymbol{x})=\sum_{j=1}^{m} c_{j} \cdot \exp \left[-\sum_{i=1}^{n} \frac{\left(x_{i}-a_{j i}\right)^{2}}{2 b_{j}^{2}}\right] .
$$

Clearly, a network just described has $3 m$ parameters. These are given by triplets $\boldsymbol{a}_{j}, b_{j}, c_{j}, j=1, \ldots m$, where $\boldsymbol{a}_{j} \in \mathcal{R}^{n}, b_{j} \in \mathcal{R}, b_{j} \neq 0$ and $c_{j} \in \mathcal{R}$.

\section{Structure learning of RBF networks}

In this section we shortly review several approaches currently used for structure learning of RBF neural networks. 
The most simple approach is the random one. This is to fix a priori a number of hidden neurons and then set their parameters in a random way. Apparently, there is no systematicness in this approach so it would be generally rejected.

A better class of approaches is a class of decremental algorithms [1]. The common strategy employed here consists of the following consecutive steps. Firstly, a broad set of candidates for hidden neurons is created and each candidate is evaluated for its suitability with respect to input/output training set. Secondly, an a priori chosen number of best evaluated candidates is taken to represent computation of hidden neurons and to determine values of respective weights. The main disadvantage of this class of algorithms is that a set of candidates typically exponentially grows with dimension of space the points of training set are taken from. This implies that for high-dimensional training data these algorithms are inapplicable because set of candidates is computationally intractable.

Common approaches capable to handle high-dimensional data are based on fuzzy clustering [2]. The idea here is to cluster training set and found clusters, representing in a numerical way some "hill like" function, transform to an analytic form of predefined type, e.g., to multidimensional Gaussians. An analytic representation then gives computation of hidden neurons and also determines values of respective weights. The main disadvantage of fuzzy clustering approaches is that an a priori chosen number of clusters has to be stated in advance.

The third class of algorithms forms incremental algorithms. These algorithms adds neurons sequentially to match as close as possible training set [3]. A general idea here is to locally fit numerical function presented by training set in each step. If there is a point which current network not "incorporate" sufficiently a new neuron is added. Typically here is mixed partial parameter learning with a structure learning. These algorithms are capable to treat high-dimensional data.

In this paper we present new incremental algorithm based on ideas issuing from neuro-fuzzy systems area.

\section{Basic idea of algorithm}

An inspiration to algorithm comes from the area of neuro-fuzzy systems [1] where three-layered RBF neural networks are used to represent fuzzy inference systems. Under this representation hidden neurons of a network are seen as particular rules of a fuzzy system. A motivation for such a representation is driven by an effort for an incorporation of neural networks' learning ability into process of creation of fuzzy systems from data. On the other hand some ideas coming from fuzzy computing can be natively applied in the area of neural networks.

One of these ideas actually gave birth to a just presented algorithm. As Kosko showed theoretically [4] particular rules of fuzzy system covers extrema of function the system is performing. With respect to fuzzy view of neural network this means that hidden neurons should in some way represent extrema of learned function. Actually, this is a main idea of algorithm which we further develop. 


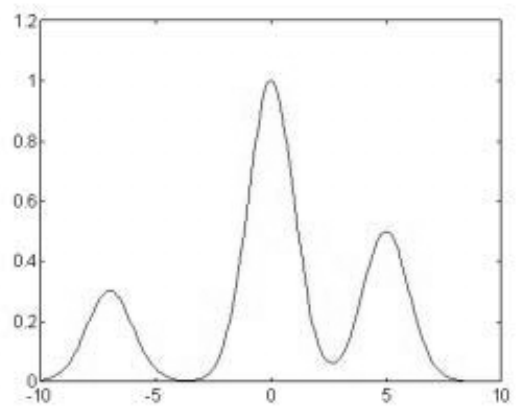

Fig. 2. A sum of three Gaussians.

We start with this one-dimensional example, see Fig. 2. A function presented here is required to be approximated by a linear combination of three Gaussians. To state explicitly parameters of these Gaussians and coefficients of the combination we can proceed in the following way. We put centers of Gaussians at points where local maxima of function are reached. Consequently, to fit function values at these points we have to multiply each Gaussian by a factor just given by the value of respective maxima. Hence maxima values gives us parameters of the linear combination. Finally, regarding width parameters, these can be set on base of a local fitting of Gaussians to approximated function. In fact the procedure presented in this example forms the skeleton of our structure learning algorithm. We now formalize this procedure in a more precise way and we also generalize it on a case of common learning data.

Regarding a generalization we have to point out that generally a function we try to approximate can also take negative values so central points has to be localized at local minima of function as well. But this situation can be transformed to only maxima case by considering in centers localization step not the original function but its absolute value. That is, we set centers at points where local maxima of absolute value of approximated function are reached.

Now, we aim on process of maxima localization. Visually, in Fig. 2., we are able to localize maxima in one instant but algorithmically this has to be formulated as iterative process assuring that linear combination of localized Gaussians gives good representation of approximated function. To fulfil this a cyclic form of algorithm is adopted where in the one step three particular actions are taken a point of maxima of absolute value of approximated function is localized, width parameter is fitted and values of approximated function are updated. Let us demonstrate these steps on the first loop of the algorithm.

In the first loop a maximum of absolute value of original function is localized. The point at which this maximum is reached gives center $a_{1}$ of the first Gaussian. Parameter of linear combination $c_{1}$ is given by the value of the original function (not its absolute value) at this point. Then local fitting is taken, see next section for details, to set up width parameter $b_{1}$. Now, after parameters of the first 
Gaussian are determined the original function is updated by subtraction of this first Gaussian from it. Values of such an updated function are then taken as a new function which undergo next iteration (the second loop) of the algorithm.

Remark here in more details to the subtraction step. It comes from an assumption that the approximated function is given by a linear combination of $m$ Gaussians. In each loop of algorithm we determine one Gaussian of this sum. This is made in such a way that after the first Gaussian's localization we subtract it from the original function (assumed to be given by a linear combination of $m$ Gaussians) and we actually obtain a new function given by a linear combination of $m-1$ Gaussians. This process is then repeated with the values of a new (obtained by subtraction) function.

Of course the task is now when we should stop the algorithm. If our assumption would be a perfectly true and sampled data were taken from a function just given by a linear combination of $m$ Gaussians then after $m$ steps subtraction yields zero function (note here explicitly that maximum of absolute value of zero function is zero) but this is not a real case. Therefore we stop the algorithm when value of maximum found is lower than some $\tau$ near to zero. Regarding setting of terminal parameter $\tau$, its value cannot be stated in an absolute form because we have to cope with generally different ranges of values of approximated functions. From this reason we state its value in a relative way as some low percentage (say $\tau=0.1$ ) of the first maximum found. This assures that linear combination of Gaussians localized by the algorithm differs maximally for $100 \tau \%$ of the first maximum found from approximated data.

This finishes detail linguistic description of the algorithm. In the following section we give the procedural transcription of the algorithm.

\section{The algorithm}

In Table 1 there is given procedural transcription of our structure learning algorithm. As it was stated purpose of the algorithm is to perform supervised structure learning task of three-layered Gaussian RBF network based on training set given as $\mathcal{T}=\left\{\left(\boldsymbol{x}_{k}, t_{k}\right)\right\}, \boldsymbol{x}_{k} \in \mathcal{R}^{n}, t_{k} \in \mathcal{R}, k=1, \ldots, N$. With respect to Table 1 we have to explain several things.

In step 01 there is made only formal renotation of original training set to its updates can be referred to.

In step 02 index $k_{1}^{*}$ of point at which at which first local maximum is reached is localized. Respective value $t_{k_{1}^{*}}$ then gives parameter of linear combination $c_{1}$.

In step 03 value of terminal parameter $\tau$ is set, e.g., $\tau=0.1$. The explanation of $\eta$ value is given further. Index $j$ refers to particular loop of the algorithm.

Steps 04-12 forms the main loop of algorithm. The loop is performed until found maximum is below $\tau$ percent of the first maximum found. In step 05 central point of $j$ th Gaussian is set. Note here that generally we work with the $n$-dimensional inputs hence center is given by a point $\boldsymbol{a} \in \mathcal{R}^{n}$. In steps 06 and 07 respective width parameter is set. We will deal with this topic in more details now. 


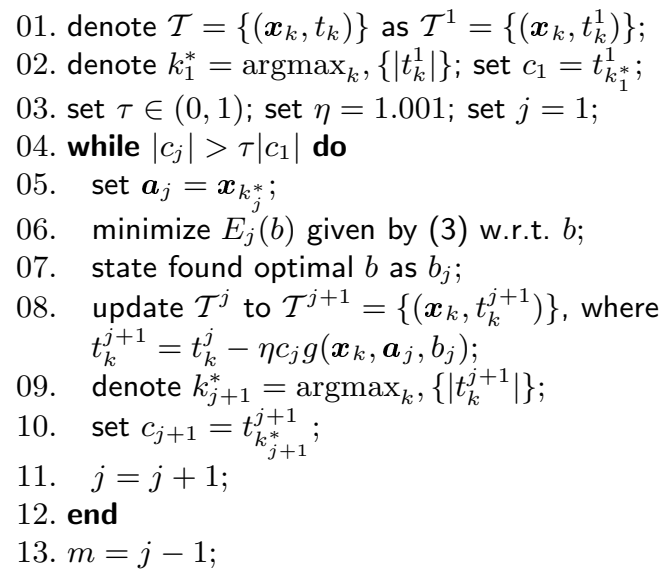

Table 1. Incremental structure learning algorithm.

As it was already mentioned width parameter should be set on base of local fitting of just identified Gaussian to approximated function. Apparently, this can be quantified in standard sense of least squares as minimization of error function $E(b)$ given as

$$
E(b)=\sum_{k=1}^{N}\left(t_{k}^{j}-\eta c_{j} g\left(\boldsymbol{a}_{j}, \boldsymbol{x}_{k}, b\right)\right) .
$$

Regarding $\eta$ parameter consider for now that $\eta=1$.

To minimize error function $E(b)$ we can use various algorithms such as golden section search, bisection method and so on [5]. But what is important that to optimization be effective we have to provide optimization algorithms by so called bracketing triplet [5]. In our case this is a triplet of points $b_{\text {left }}<b_{\text {mid }}<b_{\text {right }}$ such that $E\left(b_{\text {left }}\right)>E\left(b_{\text {mid }}\right)<E\left(b_{\text {right }}\right)$. In the following text we show that in case of our algorithm such a triplet can always be stated.

To state bracketing triplet for error function (3) with respect to $b$ we start by discussion of its properties. The first remark is that it is a function defined on intervals $(-\infty, 0) \cup(0,+\infty)$ and it is an even function which is given by the fact that $b$ occurs in Gaussians, and therefore in $E(b)$, only in its second power.

The second observation is that although originally for $b=0$ error $E$ is not defined it can be defined here. This is given by the fact that at point $b=0$ error function has discontinuity of the first order, hence we can continually define $E_{j}(0)$ by its limit at this point, i.e.,

$$
E_{j}(0)=\lim _{b \rightarrow 0} E_{j}(b)=\sum_{k_{p}}\left(t_{k_{p}}^{j}\right)^{2}+\sum_{k_{c}}\left(t_{k_{c}}^{j}-\eta c_{j}\right)^{2} .
$$

In this formula $k_{p}$ are indices of proper points of $\mathcal{T}^{j}$ which are the ones with $d^{2}\left(\boldsymbol{x}_{k_{p}}, \boldsymbol{a}_{j}\right)>0$. Indices $k_{c}$ are indices of points coinciding with central point of Gaussian, i.e., the ones with $d^{2}\left(\boldsymbol{x}_{k_{c}}, \boldsymbol{a}_{j}\right)=0$, where $d^{2}(\boldsymbol{x}, \boldsymbol{a})=\sum_{i}\left(x_{i}-a_{i}\right)^{2}$. 
In our algorithm we will consider error function enhanced on value of $b=0$. Let us now state the following assertion which proof is given in Appendix.

Lemma 1. For error function (3) there exists $b_{\max }>0$ such that inequality $E_{j}\left(2 b_{\max }\right)>E_{j}\left(b_{\max }\right)$ holds if there is at least one non-coinciding point in $\mathcal{T}^{j}$. This $b_{\max }$ is given as

$$
b_{\max }=\sqrt{\frac{d_{\max }^{2}}{2 \ln (\eta)}},
$$

where $0<d_{\text {max }}^{2}=\max _{k}\left\{d_{k}^{2}\right\}, d_{k}^{2}=\sum_{i}\left(x_{k i}-a_{j i}\right)^{2}$.

On base of this lemma we can set bracketing triplet in the following way. Firstly, we compute values $E_{j}(0)$ and $E_{j}\left(b_{\max }\right)$ and we have three cases possible.

If $E(0)<E\left(b_{\max }\right)$ we have $E\left(-b_{\max }\right)>E(0)<E\left(b_{\max }\right)$ which gives bracketing triplet as $b_{\text {left }}=-b_{\max }, b_{\text {mid }}=0, b_{\text {right }}=b_{\max }$.

If $E(0)>E\left(b_{\max }\right)$ then because we have $E\left(b_{\max }\right)<E\left(2 b_{\max }\right)$ we can set triplet as follows $b_{\text {left }}=0, b_{\text {mid }}=b_{\max }, b_{\text {right }}=2 b_{\text {max }}$.

In the case of equality $E(0)=E\left(b_{\max }\right)$ we have $E\left(b_{\max }\right)<E\left(2 b_{\max }\right)$ hence $E(0)<E\left(2 b_{\max }\right)$ which is in fact the first case. Therefore we can set triplet as $b_{\text {left }}=-2 b_{\max }, b_{\text {mid }}=0, b_{\text {right }}=2 b_{\max }$.

Having bracketing triplet set minimization of (3) can be performed by some one-dimensional optimization method [5]. But there are two minor complication. Theoretically, output of minimization can be $b=0$, in this case we set $b$ as very close to zero, e.g. $b=0.001$. The second thing is that formula (1) has good meaning only for $\eta>1$. To minimize impact of $\eta$ on sum of squares error (3) we will consider $\eta$ value very close the one, we use $\eta=1.001$. In fact above lemma justifies employment of $\eta$ parameter in error function (3).

After explanation of 06 and 07 steps of algorithm we can proceed to step 08 which states only subtraction update of approximated function.

In steps 09 and 10 we find localization of a new maxima and we set new parameter of linear combination. If found maximum fulfils condition in step 04 then loop is repeated. If not then we stop algorithm with set of $m$ localized Gaussians. This finishes explanation of algorithm.

\section{Experiments}

To demonstrate practically behavior of presented algorithm we performed the following experiment. Other experiments cannot be referred here because of lack of space. In the experiment we used sampled sinusoid function $y=\sin (x)$. Training set was formed by sampling sinusoid on interval $[0,8]$ with step 0.5 . Hence training set $\mathcal{T}=\left(x_{k}, t_{k}\right)$ was given as $x_{k}=\{0,0.5, \ldots, 0.75,8\}$ and respective $t_{k}$ as $t_{k}=\sin \left(x_{k}\right)$.

We used three structure learning algorithms - a decremental one, the one based on FCM clustering method and our incremental algorithm. In case of decremental algorithm we generates 100 candidates regulary spread on space 
$[0,8] \times[0,8] \subset \mathcal{R}^{2}$ and we chose 3 best firing. In case of FCM clustering we set number of clusters also to be 3 . For incremental clustering we used value of terminal parameter $\tau=0.2$ which gives us by three Gaussians found. Hence result in all cases gives neural network with three hidden neurons. In Table 2 we see computational error of the network after learning. The error is meant in sense of least squares, i.e., $E=\sum_{k}\left(y_{k}-o\left(x_{k}\right)\right)^{2}$.

$$
\begin{array}{c|c|c|c} 
& \text { decremental }(100 / 3) & \text { fuzzy clustering }(3) & \text { incremental }(\tau=0.2) \\
\hline \hline y=\sin (x) & 28.2(3) & 16.2(3) & 1.3(3)
\end{array}
$$

Table 2. Errors of structure learning.

From the table we see that our algorithm was able to adaptively set number of neurons (Gaussians) as 3. Output error of structure learnt network is due to adaptive fitting step less than for other two approaches hence our algorithm produce network which can be better handled by parameters learning step.

\section{Conclusion}

In the paper we propose new structure learning algorithm for three-layered Gaussian RBF networks. This algorithm does not suffer from curse of dimensionality and it behaves well with comparison with other commonly used algorithms. Presented algorithm does not require an a priori specification of number of neurons identified, however, this number is driven by the value of terminal parameter $\tau$. In the future work we aim on rejection of this parameter.

Acknolegment This work was supported by grant GACR 201/00/1489.

\section{Appendix}

Proof of Lemma 1: We start by notation remark. Denoting $d^{2}=\sum_{i}\left(x_{i}-a_{i}\right)^{2}$ we have Gaussian written as $g\left(d^{2}, b\right)$. Especially, for some given $\boldsymbol{x}_{k}$, we have $d_{k}^{2}=\sum_{i}\left(x_{k i}-a_{j i}\right)^{2}$ and Gaussian written as $g\left(d_{k}^{2}, b\right)$.

The second remark is that sometimes not all arguments for Gaussian will be written explicitly. This means that for example $g_{k}(b)$ means Gaussian with given values $\boldsymbol{a}_{j}, \boldsymbol{x}_{k}$ known from context and we are aimed only at parameter $b$ which is usually considered as variable.

To proceed, we remain two facts valid for Gaussian and one other fact for inequalities. The first fact valid for Gaussians is that

$$
\text { For fixed } d^{2}>0 \text { and for } 0<b_{1}<b_{2} \text { inequality } g\left(b_{1}\right)<g\left(b_{2}\right) \text { holds. }
$$

The second fact is that

$$
\text { For fixed } b \text { and for } 0<d_{1}^{2}<d_{2}^{2} \text { inequality } g\left(d_{1}^{2}\right)>g\left(d_{2}^{2}\right) \text { holds. }
$$


The last fact we remind is that

For $0 \leq x_{1}<x_{2}$ raising to second power retains strict inequality i.e., $x_{1}^{2}<x_{2}^{2}$.

In the following text we omit index $j$ in $E_{j}(b)$, i.e., we will write only $E(b)$. Note that overall error is given by two parts $E(b)=E_{c}+E_{p}(b)$. Constant term is given by coinciding points $\boldsymbol{x}_{k_{c}}, d^{2}\left(\boldsymbol{x}_{k_{c}}, \boldsymbol{a}\right)=0$, in form $E_{c}=\sum_{k_{c}}\left(t_{k_{c}}-\eta c\right)^{2}$. The second term is given by non-coinciding (proper) points $\boldsymbol{x}_{k_{p}}, d^{2}\left(\boldsymbol{x}_{k_{p}}, \boldsymbol{a}\right)>0$, as $E_{p}(b)=\sum_{k_{p}}\left(t_{k_{p}}-\eta c g_{k_{p}}(b)\right)^{2}$. Only this second term can be affected by $b$ parameter setting.

Now we can approach to proof itself. Let $b_{\max }$ be given in such a way that for all $k_{p}$ and for some $\eta>0$

$$
\eta|c| g_{k}\left(b_{\max }\right) \geq\left|t_{k}\right|
$$

inequality holds. We show that this assumption implies $E\left(2 b_{\max }\right)>E\left(b_{\max }\right)$.

According to $(5)$ we have $g_{k_{p}}\left(2 b_{\max }\right)>g_{k_{p}}\left(b_{\max }\right)$ for all $k_{p}$. Hence

$$
\begin{aligned}
g_{k_{p}}\left(2 b_{\max }\right) & >g_{k_{p}}\left(b_{\max }\right), \\
\eta|c| g_{k_{p}}\left(2 b_{\max }\right) & >\eta|c| g_{k_{p}}\left(b_{\max }\right), \\
\eta|c| g_{k_{p}}\left(2 b_{\max }\right)-\left|t_{k_{p}}\right| & >\eta|c| g_{k_{p}}\left(b_{\max }\right)-\left|t_{k_{p}}\right| .
\end{aligned}
$$

Since from our assumption (8) we have $\left(\eta|c| g_{k_{p}}\left(b_{\max }\right)-\left|t_{k_{p}}\right|\right) \geq 0$ we can the last equation rewrite according to (7) as

$$
\left(\eta|c| g_{k_{p}}\left(2 b_{\max }\right)-\left|t_{k_{p}}\right|\right)^{2}>\left(\eta|c| g_{k_{p}}\left(b_{\max }\right)-\left|t_{k_{p}}\right|\right)^{2} .
$$

Left side of (12) can be written as

$$
\eta^{2} c^{2} g_{k_{p}}^{2}\left(2 b_{\max }\right)-2 \eta|c| g_{k_{p}}\left(2 b_{\max }\right)\left|t_{k_{p}}\right|+t_{k_{p}}^{2}
$$

which is equal to

$$
\left(\eta c g_{k_{p}}\left(2 b_{\max }\right)-t_{k_{p}}\right)^{2}+2 \eta c g_{k_{p}}\left(2 b_{\max }\right) t_{k_{p}}-2 \eta|c| g_{k_{p}}\left(2 b_{\max }\right)\left|t_{k_{p}}\right| .
$$

Similarly, the right side of (12) can be rewritten as

$$
\left(\eta c g_{k_{p}}\left(b_{\max }\right)-t_{k_{p}}\right)^{2}+2 \eta c g_{k_{p}}\left(b_{\max }\right) t_{k_{p}}-2 \eta|c| g_{k_{p}}\left(b_{\max }\right)\left|t_{k_{p}}\right| .
$$

Let $E_{k_{p}}\left(2 b_{\max }\right)=\left(\eta c g_{k_{p}}\left(2 b_{\max }\right)-t_{k_{p}}\right)^{2}$ and $E_{k_{p}}\left(b_{\max }\right)=\left(\eta c g_{k_{p}}\left(b_{\max }\right)-t_{k_{p}}\right)^{2}$ then we have (12) in form

$$
\begin{gathered}
E_{k_{p}}\left(2 b_{\max }\right)-E_{k_{p}}\left(b_{\max }\right)> \\
2 \eta|c| g_{k_{p}}\left(2 b_{\max }\right)\left|t_{k_{p}}\right|-2 \eta c g_{k_{p}}\left(2 b_{\max }\right) t_{k_{p}}+2 \eta c g_{k_{p}}\left(b_{\max }\right) t_{k_{p}}-2 \eta|c| g_{k_{p}}\left(b_{\max }\right)\left|t_{k_{p}}\right|
\end{gathered}
$$

Term (16) can be rewritten as

$$
2 \eta g_{k_{p}}\left(2 b_{\max }\right)\left(|c|\left|t_{k_{p}}\right|-c t_{k_{p}}\right)+2 \eta g_{k_{p}}\left(b_{\max }\right)\left(c t_{k_{p}}-|c|\left|t_{k_{p}}\right|\right)
$$


which is

$$
2 \eta\left(\left|c t_{k_{p}}\right|-c t_{k_{p}}\right)\left(g_{k_{p}}\left(2 b_{\max }\right)-g_{k_{p}}\left(b_{\max }\right)\right) .
$$

Since $|x|-x \geq 0$ for all $x \in \mathcal{R}$ and $\left(g_{k_{p}}\left(2 b_{\max }\right)-g_{k_{p}}\left(b_{\max }\right)\right)>0$ according to (5) we have term $(18) \geq 0$. That is, for all $k_{p}$ holds $E_{k_{p}}\left(2 b_{\max }\right)-E_{k_{p}}\left(b_{\max }\right)>0$ which gives, summing through $k_{p}, E_{p}\left(2 b_{\max }\right)-E_{p}\left(b_{\max }\right)>0$. Since we assumed that there is at least one proper point we have with respect to overall error $E(b)=E_{c}+E_{p}(b)$

$$
E\left(2 b_{\max }\right)>E\left(b_{\max }\right) .
$$

Now, we aim on task how to set $b_{\max }$ to condition (8) holds. Since for $c$ inequality $|c| \geq\left|t_{k}\right|$ holds for all $k$, see point 02 or 09 of Table 1 , a setting of $b_{\max }$ in such a way that for all $k$

$$
\eta|c| g_{k}\left(b_{\max }\right) \geq|c|
$$

solves the problem. Clearly (20) can be written as

$$
g_{k}\left(b_{\max }\right) \geq 1 / \eta
$$

Considering $0<d_{\max }^{2}=\max _{k}\left\{d_{k}^{2}\right\}, d_{k}^{2} \leq d_{\text {max }}^{2}$, we have according to (6) for all $k, g_{k}\left(b_{\max }\right)=g\left(d_{k}^{2}, b_{\max }\right) \geq g\left(d_{\max }^{2}, b_{\max }\right)$. Due to this inequality inequality (21) is valid when $b_{\max }$ is set in such a way that

$$
g\left(d_{\max }^{2}, b_{\max }\right)=1 / \eta
$$

This gives for $b_{\max }$ expression

$$
\frac{d_{\max }^{2}}{2 b_{\max }^{2}}=-\ln \left(\frac{1}{\eta}\right) \quad \text { and therefore } \quad b_{\max }=\sqrt{\frac{d_{\max }^{2}}{2 \ln (\eta)}} .
$$

\section{References}

1. Nauck D., Klawonn F., Kruse R.: Foundations of Neuro-Fuzzy Systems. John Wiley \& Sons, 1997

2. Höppner F., et al.: Fuzzy cluster analysis. John Wiley \& Sons, 1999

3. Cho B.C., Wang B.H.,: Radial basis function based adaptive fuzzy systems and their applications to system identification and prediction. Fuzzy sets and systems, 83, no.2, (1996) 325-339

4. Kosko B.: Optimal fuzzy rules cover extrema. Int. J. Intell Syst., 10, no.2, (1995) 249-255

5. Press W.H., Teukolsky S.A., Vetterling W.T, Flannery B.P., Numerical Recipes in C, The Art of Scientific Computing, Second Edition, Cambridge University Press, 1992; internet version is available at http://www.nr.com 